PROCEEDINGS OF THE

AMERICAN MATHEMATICAL SOCIETY

Volume 137, Number 4, April 2009, Pages 1339-1345

S 0002-9939(08)09726-8

Article electronically published on December 3, 2008

\title{
ON THE LOCALIZATION PRINCIPLE FOR THE AUTOMORPHISMS OF PSEUDOELLIPSOIDS
}

\author{
MARIO LANDUCCI AND ANDREA SPIRO
}

(Communicated by Mei-Chi Shaw)

\begin{abstract}
We show that Alexander's extendibility theorem for a local automorphism of the unit ball is valid also for a local automorphism $f$ of a pseudoellipsoid $\mathcal{E}_{\left(p_{1}, \ldots, p_{k}\right)}^{n} \stackrel{\text { def }}{=}\left\{z \in \mathbb{C}^{n}: \sum_{j=1}^{n-k}\left|z_{j}\right|^{2}+\left|z_{n-k+1}\right|^{2 p_{1}}+\cdots+\left|z_{n}\right|^{2 p_{k}}<1\right\}$, provided that $f$ is defined on a region $\mathcal{U} \subset \mathcal{E}_{(p)}^{n}$ such that: i) $\partial \mathcal{U} \cap \partial \mathcal{E}_{(p)}^{n}$ contains an open set of strongly pseudoconvex points; ii) $\mathcal{U} \cap\left\{z_{i}=0\right\} \neq \emptyset$ for any $n-k+1 \leq i \leq n$. By the counterexamples we exhibit, such hypotheses can be considered as optimal.
\end{abstract}

\section{INTRODUCTION}

For a given $k$-tuple of integers $p=\left(p_{1}, \ldots, p_{k}\right)$, with each $p_{\ell} \geq 2$, let us denote by $\mathcal{E}_{\left(p_{1}, \ldots, p_{k}\right)}^{n}$ (or, more simply, $\left.\mathcal{E}_{(p)}^{n}\right)$ the pseudoellipsoid in $\mathbb{C}^{n}$ defined by

$$
\mathcal{E}_{\left(p_{1}, \ldots, p_{k}\right)}^{n} \stackrel{\text { def }}{=}\left\{z \in \mathbb{C}^{n}: \sum_{j=1}^{n-k}\left|z_{j}\right|^{2}+\left|z_{n-k+1}\right|^{2 p_{1}}+\cdots+\left|z_{n}\right|^{2 p_{k}}<1\right\} .
$$

When $k=0$, we assume $\mathcal{E}_{(p)}^{n}$ to be the unit ball $B^{n}=\left\{z \in \mathbb{C}^{n}:|z|<1\right\}$. Now, let us consider the following definition.

Definition 1.1. We define a local automorphism of $\mathcal{E}_{(p)}^{n}$ to be any biholomorphic map $f: \mathcal{U}_{1} \subset \mathcal{E}_{(p)}^{n} \rightarrow \mathcal{U}_{2} \subset \mathcal{E}_{(p)}^{n}$ between two connected open subsets of $\mathcal{E}_{(p)}^{n}$ such that:

a) each of the intersections $\partial \mathcal{U}_{i} \cap \partial \mathcal{E}_{(p)}^{n}, i=1,2$, contains a boundary open set $\Gamma_{i} \subset \partial \mathcal{E}_{(p)}^{n}$

b) there exists at least one sequence $\left\{x_{k}\right\} \subset \mathcal{U}_{1}$ which converges to a point $x_{o} \in \Gamma_{1}$, which is not a limit point of $\partial \mathcal{U}_{1} \cap \mathcal{E}_{(p)}^{n}$, and so that $\left\{f\left(x_{k}\right)\right\}$ converges to a point $\hat{x}_{o} \in \Gamma_{2}$, which is not a limit point of $\partial \mathcal{U}_{2} \cap \mathcal{E}_{(p)}^{n}$.

We say that a local automorphism $f: \mathcal{U}_{1} \subset \mathcal{E}_{(p)}^{n} \rightarrow \mathcal{U}_{2} \subset \mathcal{E}_{(p)}^{n}$ extends to a global automorphism of $\mathcal{E}_{(p)}^{n}$ if there exists some $F \in \operatorname{Aut}\left(\mathcal{E}_{(p)}^{n}\right)$ such that $\left.F\right|_{\mathcal{U}_{1} \cap \mathcal{E}_{(p)}^{n}} ^{n}=$ $\left.f\right|_{\mathcal{U}_{1} \cap \mathcal{E}_{(p)}^{n}} \cdot$

By a celebrated theorem of Alexander and its generalization obtained by Rudin ([Al, $[\mathrm{Ru}]$ ), when $\mathcal{E}_{(p)}^{n}=B^{n}$, any local automorphism extends to a global one.

Received by the editors June 25, 2007, and, in revised form, February 17, 2008.

2000 Mathematics Subject Classification. Primary 32H12, 32H02, 32H35.

Key words and phrases. Alexander theorem, pseudoellipsoids, localization principle.

(C)2008 American Mathematical Society Reverts to public domain 28 years from publication 
This crucial extendibility result is often referred to as the localization principle for the automorphisms of $B^{n}$, and it has been extended or established under different but similar hypotheses for a wide class of domains besides the unit balls (see e.g. [DS, Pi, Pi1]). On the other hand, even if it is known that the pseudoellipsoids $\mathcal{E}_{(p)}^{n}$ share many useful properties with $B^{n}$ for what concerns the global automorphisms and the proper holomorphic maps (see for instance $\mathrm{We}, \mathrm{La}, \mathrm{LS}, \mathrm{DS}$ ), some simple examples show that Alexander's theorem cannot be true in full generality for a pseudoellipsoid $\mathcal{E}_{(p)}^{n}$ different from $B^{n}$ (see e.g. Example 3.4 below).

Nonetheless, for each $\mathcal{E}_{(p)}^{n}$, it is possible to determine, precisely and in an efficient way, the class of local automorphisms that can be extended to global ones. In this short note we give a characterization of such local automorphisms by means of the following generalization of Alexander's theorem.

Theorem 1.2. Let $f: \mathcal{U}_{1} \subset \mathcal{E}_{(p)}^{n} \rightarrow \mathcal{U}_{2} \subset \mathcal{E}_{(p)}^{n}$ be a local automorphism of a pseudoellipsoid $\mathcal{E}_{(p)}^{n}$, with $p=\left(p_{1}, \ldots, p_{k}\right)$, and satisfying the following two conditions:

i) there exists a sequence $\left\{x_{i}\right\}$ as in (b) of Definition 1.1, whose limit point $x_{o} \in \partial \mathcal{E}_{(p)}$ is Levi non-degenerate;

ii) for any $n-k+1 \leq i \leq n$, the intersection $\mathcal{U}_{1} \cap\left\{z_{i}=0\right\}$ is not empty.

Then $f$ extends to a global automorphism $f \in \operatorname{Aut}\left(\mathcal{E}_{(p)}^{n}\right)$.

We point out that the set $\partial \mathcal{E}_{(p)}^{n} \cap \bigcup_{i=n-k+1}^{n}\left\{z_{i}=0\right\}$ coincides with the set of points of Levi degeneracy of $\partial \mathcal{E}_{(p)}^{n}$. So, Theorem 1.2 can be roughly stated by saying that $f$ is globally extendible as soon as it admits a holomorphic extension to some open subset $\mathcal{U} \subset \mathcal{E}_{(p)}^{n}$, which intersects each of the hyperplanes containing the Levi degeneracy set of $\partial \mathcal{E}_{(p)}^{n}$ and, at the same time, the boundary $\partial \mathcal{U}$ contains an open set of strongly pseudoconvex points of $\partial \mathcal{E}_{(p)}^{n}$.

From Example 3.4, it will be clear that such hypotheses can be considered as optimal.

The properties of the pseudoellipsoid used in the proof are basically just two: (1) It admits a finite ramified covering over the unit ball; (2) Its automorphisms are "lifts" of the automorphisms of the unit ball that preserve the singular values of the covering. Since (2) is a consequence of (1), it is reasonable to expect that a similar result should be true for any arbitrary ramified covering of the unit ball.

About this more general problem, we refer to KLS, KS for what concerns the classification of the domains in $\mathbb{C}^{2}$ that admit a ramified holomorphic covering over $B^{2}$.

\section{ON THE AUTOMORPHISMS OF THE UNIT BALL}

First of all, we need to recall some basic facts on the automorphisms of the unit ball. Let us denote by $\hat{\imath}: \mathbb{C}^{n} \rightarrow \mathbb{C} P^{n}$ the canonical embedding

$$
\hat{\imath}: \mathbb{C}^{n} \rightarrow \mathbb{C} P^{n}, \quad \hat{\imath}(z)=\left[\begin{array}{c}
z_{1} \\
\vdots \\
z_{n} \\
1
\end{array}\right]
$$

and let $\hat{\mathbb{C}}^{n}=\hat{\imath}\left(\mathbb{C}^{n}\right)=\mathbb{C} P^{n} \backslash\left\{[w]: w_{n+1}=0\right\}$. We recall that, via the embedding, $B^{n}$ corresponds to the projective open set $\hat{B}^{n}=\left\{[w] \in \mathbb{C} P^{n}:\langle w, w\rangle<0\right\}$, 
where we denote by $\langle$,$\rangle the pseudo-Hermitian inner product on \mathbb{C}^{n+1}$ defined by

$$
\langle w, z\rangle=\bar{w}^{t} \cdot I_{n, 1} \cdot z, \quad \text { where } I_{n, 1} \stackrel{\text { def }}{=}\left(\begin{array}{cc}
I_{n} & 0 \\
0 & -1
\end{array}\right) \text {. }
$$

It is also known that a holomorphic map $F: B^{n} \rightarrow B^{n}$ is an automorphism of $B^{n}$ if and only if the corresponding map $\hat{F}=\hat{\imath} \circ F \circ \hat{\imath}^{-1}: \hat{B}^{n} \rightarrow \hat{B}^{n}$ is a projective linear transformation which preserves the quadric $\partial \hat{B}^{n}=\{[w]:\langle w, w\rangle=0\}$ (see e.g. $\mathrm{Ve})$. This means that $\hat{F}$ is of the form

$$
\hat{F}([z])=[\mathbb{A} \cdot z]
$$

where $\mathbb{A}$ is a matrix in $\mathrm{SU}_{n, 1}$, i.e. such that $\overline{\mathbb{A}}^{t} I_{n, 1} \mathbb{A}=I_{n, 1}$ and with $\operatorname{det} \mathbb{A}=1$.

The correspondence $F \mapsto \hat{F}=\hat{\imath} \circ F \circ \hat{\imath}^{-1}$ gives an isomorphism between $\operatorname{Aut}\left(B_{n}\right)$ and $\mathrm{SU}_{n, 1} / K$, where $K=\left\{e^{i \frac{2 \pi k}{n+1}} I_{n+1}, 0 \leq k \leq n\right\}$.

The identification of the elements of $\operatorname{Aut}\left(B^{n}\right)$ with the corresponding projective linear transformations is often quite useful, for instance in order to establish the following fact (see also $\mathrm{We}, \S 6)$.

Lemma 2.1. Let $F=\left(F_{1}, \ldots, F_{n}\right) \in \operatorname{Aut}\left(B^{n}\right)$ be an automorphism such that

$$
F\left(B^{n} \cap\left\{z_{i}=0\right\}\right) \subset\left\{z_{i}=0\right\}
$$

for all $n-k+1 \leq i \leq n$. Then the components $F_{i}$ are of the following form:

$$
\begin{aligned}
& F_{j}(z)=\frac{\sum_{\ell=1}^{n-k} A_{j}^{\ell} z_{\ell}+b_{j}}{\sum_{\ell=1}^{n-k} c^{\ell} z_{\ell}+d}, \quad \text { for } 1 \leq j \leq n-k, \\
& F_{j}(z)=e^{i \theta_{j}} z_{j} \frac{1}{\sum_{\ell=1}^{n-k} c^{\ell} z_{\ell}+d}, \quad \text { for } n-k+1 \leq j \leq n,
\end{aligned}
$$

for some $\theta_{j} \in \mathbb{R}$ and where $A=\left(A_{j}^{i}\right), b=\left(b_{j}\right), c=\left(c^{\ell}\right)$ and $d$ are such that $\left(\begin{array}{ll}A & b \\ c & d\end{array}\right) \in \mathrm{SU}_{n-k, 1}$. In particular, the maps $F_{j}, 1 \leq j \leq n-k$, coincide with the components of an element of $\operatorname{Aut}\left(B^{n-k}\right)$, while $\sum_{j=1}^{n-k} c^{j} z_{j}+d \neq 0$ for any $z \in B^{n}$.

Proof. By hypothesis, the corresponding automorphism $\hat{F}=\hat{\imath} \circ F \circ \hat{\imath}^{-1} \in \operatorname{Aut}\left(\hat{B}^{n}\right)$ maps all hyperplanes $H_{i}=\left\{[w] \in \mathbb{C} P^{n}: w_{i}=0\right\}$ into themselves and hence fixes their poles relative to the quadric $\partial \hat{B}^{n}$, i.e. fixes all the points

$$
\left[e_{i}\right]=\left[0: \ldots: 0:{ }_{i-t h \text { place }}: 0: \ldots: 0\right], \quad n-k+1 \leq i \leq n .
$$

This implies that the matrix $\mathbb{A}$ which determines the projective transformation $\hat{F}$ is of the form

$$
\mathbb{A}=\left(\begin{array}{ccccc}
A & 0 & \ldots & 0 & b \\
0 & e^{i \theta_{n-k+1}} & & 0 & 0 \\
\vdots & & & \ddots & \\
0 & 0 & & e^{i \theta_{n}} & 0 \\
c & 0 & \ldots & 0 & d
\end{array}\right)
$$

where $A, b, c$ and $d$ are such that $\mathbb{A}^{\prime} \stackrel{\text { def }}{=}\left(\begin{array}{ll}A & b \\ c & d\end{array}\right)$ belongs to $\mathrm{SU}_{n-k, 1}$. From this, (2.4) and (2.5) follow immediately. The last claim follows from the fact that the value $\sum_{\ell=1}^{n-k} c^{\ell} z_{\ell}+d$ is the last homogeneous coordinate of the element 
$\left[\mathbb{A}^{\prime} \cdot\left(z_{1}: \ldots: z_{n-k}: 1\right)\right] \in \mathbb{C} P^{n-k}$ and it is clearly different from 0 , since the map $[w] \mapsto\left[\mathbb{A}^{\prime} \cdot w\right]$ is an automorphism of $\hat{B}^{n-k} \subset \mathbb{C} P^{n-k} \backslash\left\{w_{n-k+1} \neq 0\right\}$.

\section{Proof of Theorem 1.2}

First of all, we need to introduce the following notation. For any $p=\left(p_{1}, \ldots, p_{k}\right)$, we will use the symbol $\pi^{(p)}$ to denote the map

$$
\pi^{(p)}: \mathbb{C}^{n} \rightarrow \mathbb{C}^{n}, \quad \pi^{(p)}(z)=\left(z_{1}, \ldots, z_{n-k}, z_{n-k+1}^{p_{1}}, \ldots, z_{n}^{p_{k}}\right) .
$$

We recall that the restriction $\left.\pi^{(p)}\right|_{\mathcal{E}_{(p)}^{n}}$ gives a proper holomorphic map $\pi^{(p)}$ : $\mathcal{E}_{(p)}^{n} \longrightarrow B^{n}$.

Secondly, we need to recall a useful theorem by Forstneric and Rosay ([FR]). Given a domain $D \subset \mathbb{C}^{n}$, we say that a boundary point $z_{o} \in \partial D$ satisfies the condition $(P)$ if:

- $\partial D$ is of class $\mathcal{C}^{1+\varepsilon}$ near $z_{o}$ for some $\varepsilon>0$;

- there exist a continuous negative plurisubharmonic function $\rho$ on $D$ and a neighborhood $\mathcal{U}$ of $z_{o}$ so that $\rho(z) \geq-c d(z, \partial D)$ at all points of $\mathcal{U} \cap D$ for some constant $c>0$.

Theorem 1.1 and some related remarks of [FR] can be summarized as follows.

Theorem 3.1. Let $h: D \rightarrow D^{\prime}$ be a proper holomorphic map between two domains of $\mathbb{C}^{n}$ and let $z_{o} \in \partial D$ be a point that satisfies the condition $(P)$.

If there exists a sequence $\left\{z_{j}\right\} \subset D$ so that $\lim _{j \rightarrow \infty} z_{j}=z_{o}$ and $\lim _{j \rightarrow \infty} h\left(z_{j}\right)=$ $\hat{z}_{o}$ for some $\hat{z}_{o} \in \partial D^{\prime}$ at which $\partial D^{\prime}$ is $\mathcal{C}^{2}$ and strictly pseudoconvex, then $h$ extends continuously to all points of a neighborhood $\mathcal{V}$ of $z_{o}$ in $\bar{D}$.

We may now prove the following lemma.

Lemma 3.2. Let $f: \mathcal{U}_{1} \subset \mathcal{E}_{(p)}^{n} \rightarrow \mathcal{U}_{2} \subset \mathcal{E}_{(p)}^{n}$ be a local automorphism of a pseudoellipsoid $\mathcal{E}_{(p)}^{n}$ with $p=\left(p_{1}, \ldots, p_{k}\right)$ and assume that

i) there exists a sequence $\left\{x_{i}\right\}$ as in (b) of Definition 1.1, whose limit point $x_{o} \in \partial \mathcal{E}_{(p)}$ is Levi non-degenerate;

ii) for any $n-k+1 \leq i \leq n$, the intersection $\mathcal{U}_{1} \cap\left\{z_{i}=0\right\}$ is not empty.

Then, up to composition with a coordinate permutation,

$$
\left(z_{1}, \ldots, z_{n}\right) \mapsto\left(z_{\sigma(1)}, \ldots, z_{\sigma(n)}\right),
$$

the map $f$ sends the points of the hyperplane $\left\{z_{i}=0\right\}$ into the same hyperplane for any $n-k+1 \leq i \leq n$.

Proof. In all the following we will use the symbols $\Gamma_{i}, x_{o}$ and $\hat{x}_{o}$ with the same meaning as in Definition 1.1

First of all, notice that $\hat{x}_{o} \in \Gamma_{2} \subset \partial \mathcal{U}_{2}$ satisfies the condition (P) and hence, by Theorem 3.1. for any sufficiently small ball $B_{\varepsilon}\left(\hat{x}_{o}\right)$, centered at $\hat{x}_{o}$ and of radius $\varepsilon$, the holomorphic map $f^{-1}: \mathcal{U}_{2} \rightarrow \mathcal{U}_{1}$ extends continuously to all points of $\overline{B_{\varepsilon}\left(\hat{x}_{o}\right)} \cap \Gamma_{2}$. In particular, we may assume that $f^{-1}\left(\overline{B_{\varepsilon}\left(\hat{x}_{o}\right) \cap \Gamma_{2}}\right)$ is contained in a neighborhood of $x_{o}=f^{-1}\left(\hat{x}_{o}\right)$ in $\Gamma_{1}$ in which there are no Levi degenerate points.

Pick a Levi non-degenerate point $\hat{x}_{o}^{\prime} \in \overline{B_{\varepsilon}\left(\hat{x}_{o}\right)} \cap \Gamma_{2}$ and consider a sequence $\left\{\hat{x}_{k}^{\prime}\right\} \subset \overline{B_{\varepsilon}\left(\hat{x}_{o}\right)} \cap \mathcal{U}_{2}$ which converges to $\hat{x}_{o}^{\prime}$. By construction, the sequence $\left\{x_{k}^{\prime}=\right.$ $\left.f^{-1}\left(\hat{x}_{k}^{\prime}\right)\right\} \subset \mathcal{U}_{1}$ converges to the Levi non-degenerate point $x_{o}^{\prime}=f^{-1}\left(\hat{x}_{o}^{\prime}\right) \in \Gamma_{1}$. It follows that, replacing $x_{o}$ by $x_{o}^{\prime}$ and $\hat{x}_{o}$ by $\hat{x}_{o}^{\prime}$ and by Theorem 3.1 applied to 
$f$ and $f^{-1}$, there is no loss of generality if we assume that $x_{o}$ and $\hat{x}_{o}$ are both Levi non-degenerate and that, for any sufficiently small $\varepsilon_{1}>0$, the map $f$ extends continuously to a map

$$
f: \mathcal{U}_{1} \cup\left(\overline{B_{\varepsilon_{1}}\left(x_{o}\right)} \cap \Gamma_{1}\right) \rightarrow \mathcal{U}_{2} \cup\left(B_{\varepsilon}\left(\hat{x}_{o}\right) \cap \Gamma_{2}\right),
$$

which is a homeomorphism onto its image.

Since the complex Jacobian matrices $\left.J \pi^{(p)}\right|_{x_{o}}$ and $\left.J \pi^{(p)}\right|_{\hat{x}_{o}}$ are of maximal rank (recall that $x_{o}$ and $\hat{x}_{o} \in \partial \mathcal{E}_{(p)}^{n}$ are both Levi non-degenerate), from the fact that $x_{o}$ is not a limit point of $\partial \mathcal{U}_{1} \cap \mathcal{E}_{(p)}^{n}$ and by the continuity of $f$ and $f^{-1}$ around $x_{o}$ and $\hat{x}_{o}$, respectively, we may choose $\varepsilon_{1}$ and $\varepsilon_{2}$ so that:

a) $\left.\pi^{(p)}\right|_{B_{\varepsilon_{1}}\left(x_{o}\right)}$ and $\left.\pi^{(p)}\right|_{B_{\varepsilon_{2}}\left(\hat{x}_{o}\right)}$ are both biholomorphisms onto their images;

b) $\overline{f\left(B_{\varepsilon_{1}}\left(x_{o}\right) \cap \mathcal{U}_{1}\right)} \subset B_{\varepsilon_{2}}\left(\hat{x}_{o}\right)$ and $\left.f\right|_{B_{\varepsilon_{1}}\left(x_{o}\right) \cap \mathcal{U}_{1}}$ extends to a homeomorphism between $\overline{B_{\varepsilon_{1}}\left(x_{o}\right) \cap \mathcal{U}_{1}}$ and $\overline{f\left(B_{\varepsilon_{1}}\left(x_{o}\right) \cap \mathcal{U}_{1}\right)}$ which induces a homeomorphism between $B_{\varepsilon_{1}}\left(x_{o}\right) \cap \Gamma_{1}$ and $f\left(B_{\varepsilon_{1}}\left(x_{o}\right) \cap \Gamma_{1}\right) \subset \Gamma_{2}$.

Notice that, by definition, $x_{o}$ is not a limit point of $\partial\left(B_{\varepsilon_{1}}\left(x_{o}\right) \cap \mathcal{U}_{1}\right) \cap \mathcal{E}_{(p)}^{n}$ and, by (b), $\hat{x}_{o}$ is not a limit point of $\partial f\left(B_{\varepsilon_{1}}\left(x_{o}\right) \cap \mathcal{U}_{1}\right) \cap \mathcal{E}_{(p)}^{n}$. So, if we set

$$
\mathcal{U}_{1}^{\prime} \stackrel{\text { def }}{=} B_{\varepsilon_{1}}\left(x_{o}\right) \cap \mathcal{U}_{1}, \quad \mathcal{U}_{2}^{\prime} \stackrel{\text { def }}{=} f\left(\mathcal{U}_{1}^{\prime}\right) \subset B_{\varepsilon_{2}}\left(\hat{x}_{o}\right), \quad \mathcal{V}_{i} \stackrel{\text { def }}{=} \pi^{(p)}\left(\mathcal{U}_{i}^{\prime}\right) \quad i=1,2,
$$

then the maps

and

$$
\left.f\right|_{\mathcal{U}_{1}^{\prime}}: \mathcal{U}_{1}^{\prime} \rightarrow \mathcal{U}_{2}^{\prime}
$$

$$
\tilde{f}=\left.\pi^{(p)} \circ f \circ \pi^{(p)-1}\right|_{\mathcal{V}_{1}}: \mathcal{V}_{1} \subset B^{n} \longrightarrow \mathcal{V}_{2} \subset B^{n}
$$

are local automorphisms of $\mathcal{E}_{(p)}^{n}$ and of the unit ball, respectively.

By Rudin's generalization of Alexander's theorem ( $[\mathrm{Ru}]$ ), this implies that $\tilde{f}$ extends to a global automorphism of $B^{n}$, which we denote by $\tilde{f}$ as well. By construction, for any $z \in \mathcal{U}_{1}^{\prime}=\pi^{(p)-1}\left(\mathcal{V}_{1}\right)$, we have

$$
\tilde{f} \circ \pi^{(p)}(z)=\pi^{(p)} \circ f(z),
$$

but since both sides have a holomorphic extension on $\mathcal{U}_{1}$, we get that (3.2) must be true also for any $z$ in such a larger set.

In particular,

$$
\left.\left.J(\tilde{f})\right|_{\pi^{(p)}(z)} \cdot J\left(\pi^{(p)}\right)\right|_{z}=\left.\left.J\left(\pi^{(p)}\right)\right|_{f(z)} \cdot J(f)\right|_{z}, \quad \text { for any } z \in \mathcal{U}_{1} .
$$

Since for any $z \in \mathcal{U}_{1}$, det $\left.J(f)\right|_{z} \neq 0$ and

$$
\left\{\left.J\left(\pi^{(p)}\right)\right|_{z}=0\right\}=\bigcup_{i=n-k+1}^{n}\left\{z_{i}=0\right\}
$$

equality (3.3) implies that, for any $n-k+1 \leq i \leq n$ and $z \in \mathcal{U}_{1} \cap\left\{z_{i}=0\right\}$, the value of $\left.J\left(\pi^{(p)}\right)\right|_{f(z)}$ is 0 . By (3.4), this means that $f\left(\mathcal{U}_{1} \cap\left\{z_{i}=0\right\}\right)$ is contained in the union $\bigcup_{j=n-k+1}^{n}\left\{z_{j}=0\right\}$. Indeed, it is contained in exactly one of the hyperplanes $\left\{z_{j}=0\right\}$, because $f$ is a biholomorphism and consequently $f\left(\mathcal{U}_{1} \cap\left\{z_{i}=0\right\}\right)$ is an irreducible analytic variety. From this the conclusion follows.

We proceed by defining a rule that associates an automorphism of $B^{n}$ with any local automorphism of a pseudoellipsoid (see also [We, $\S 6$ ). Given a local automorphism $f: \mathcal{U} \rightarrow \mathbb{C}^{n}$ of $\mathcal{E}_{(p)}^{n}$, pick a point $x_{o} \in \mathcal{U} \cap \partial \mathcal{E}_{(p)}^{n}$ for which (b) of 
Definition 1.1 holds and determine a small ball $B_{\varepsilon}\left(x_{o}\right)$ centered in $x_{o}$ as in the proof of the previous lemma. Then, we denote by $\tilde{f} \in \operatorname{Aut}\left(B^{n}\right)$ the global automorphism of the unit ball that extends $\left.\tilde{f} \stackrel{\text { def }}{=} \pi^{(p)} \circ f \circ \pi^{(p)-1}\right|_{\pi^{(p)}(\mathcal{V})}$, with $\mathcal{V} \stackrel{\text { def }}{=} B_{\varepsilon}\left(x_{o}\right) \cap \mathcal{E}_{(p)}^{n}$. By the identity principle of the holomorphic maps, such an automorphism $\tilde{f}$ depends only on $f$ and will be called the (global) automorphism of $B^{n}$ associated with $f$.

With the help of such a correspondence, we may state the following criterion for extendibility of local automorphisms.

Proposition 3.3. A local automorphism $f: \mathcal{U}_{1} \subset \mathcal{E}_{(p)}^{n} \rightarrow \mathcal{U}_{2} \subset \mathcal{E}_{(p)}^{n}$ of a pseudoellipsoid $\mathcal{E}_{(p)}^{n}, p=\left(p_{1}, \ldots, p_{k}\right)$, extends to a global automorphism $f \in \operatorname{Aut}\left(\mathcal{E}_{(p)}^{n}\right)$ if and only if its associated automorphism $\tilde{f} \in \operatorname{Aut}\left(B^{n}\right)$ satisfies (2.3) for any $n-k+1 \leq i \leq n$, up to composition with a permutation of those coordinates $z_{n-k+j}$, for which the integers $p_{j}$ are of the same value.

Proof. Assume that the local automorphism $f: \mathcal{U} \rightarrow \mathbb{C}^{n}$ extends to a global automorphism $f \in \operatorname{Aut}\left(\mathcal{E}_{(p)}^{n}\right)$ and recall that, by construction, the associated automorphism $\tilde{f} \in \operatorname{Aut}\left(B^{n}\right)$ satisfies (3.2) at all points where $f$ is defined (in this case, at all points of $\left.\mathcal{E}_{(p)}^{n}\right)$. Then, by Lemma 3.2 and the fact that $\pi^{(p)}\left(\mathcal{E}_{(p)}^{n} \cap\left\{z_{i}=0\right\}\right)=$ $B^{n} \cap\left\{z_{i}=0\right\}$, the equality (3.3) implies that, up to a suitable permutation of coordinates, $\tilde{f}$ satisfies (2.3) for any $n-k+1 \leq i \leq n$.

Conversely, assume that $f=\left(f_{1}, \ldots, f_{n}\right): \mathcal{U}_{1} \subset \mathcal{E}_{(p)}^{n} \rightarrow \mathcal{U}_{2} \subset \mathcal{E}_{(p)}^{n}$ is a local automorphism of $\mathcal{E}_{(p)}^{n}$ such that (up to a suitable permutation of coordinates) the associated automorphism $\left.\tilde{f}=\left(\tilde{f}_{1}, \ldots, \tilde{f}_{n}\right) \in \operatorname{Aut}\left(B^{n}\right)\right)$ satisfies (2.3) for any $n-$ $k+1 \leq i \leq n$. From (2.4), (2.5) and (3.2), it follows that the components $f_{j}$ of $f$ are of the form

$$
\begin{aligned}
f_{j}(z) & =\frac{\sum_{\ell=1}^{n-k} A_{j}^{\ell} z_{\ell}+b_{j}}{\sum_{\ell=1}^{n-k} c^{\ell} z_{\ell}+d}, \quad \text { for } 1 \leq j \leq n-k, \\
f_{n-k+j}(z) & =e^{i \theta_{j}} z_{j} \frac{1}{\left(\sum_{\ell=1}^{n-k} c^{\ell} z_{\ell}+d\right)^{\frac{1}{p_{j}}}}, \quad \text { for } 1 \leq j \leq k,
\end{aligned}
$$

for some fixed definitions of the $p_{j}$-th roots $w \mapsto w^{\frac{1}{p_{j}}}$.

From (3.5) and (3.6) it follows immediately that $f$ coincides with a globally defined automorphism of $\mathcal{E}_{(p)}^{n}$ (for the general expressions of the elements in $\operatorname{Aut}\left(\mathcal{E}_{(p)}^{n}\right)$, see $\mathrm{We}, \mathrm{La}]$ ).

Now, Theorem 1.2 follows almost immediately. In fact, if $f: \mathcal{U}_{1} \subset \mathcal{E}_{(p)}^{n} \rightarrow \mathcal{U}_{2} \subset$ $\mathcal{E}_{(p)}^{n}$ is a local automorphism satisfying the hypotheses of the theorem, by Lemma 3.2 and (3.2), the associated automorphism $\tilde{f} \in \operatorname{Aut}\left(B^{n}\right)$ satisfies the hypotheses of Proposition 3.3 and the claim follows.

We conclude with the following simple construction of non-extendible local automorphisms of pseudoellipsoids.

Example 3.4. Let $\tilde{f} \in \operatorname{Aut}\left(B^{n}\right)$ be an automorphism which does not satisfy (2.3) for some $n-k+1 \leq j \leq n$. Pick a point $w_{o} \in \partial B \cap\left\{\prod_{j=n-k+1}^{n} z_{j} \neq 0\right\}$ so that also its image $\tilde{f}\left(w_{o}\right)$ is in $\partial B \cap\left\{\prod_{j=n-k+1}^{n} z_{j} \neq 0\right\}$. Then, let $z_{o} \in \partial \mathcal{E}_{(p)}^{n}$ so that $\pi^{(p)}\left(z_{o}\right)=w_{o}$ and consider a connected neighborhood $\mathcal{U}$ of $z_{o}$ with the 
following two properties: a) $\pi^{(p)} \mid \mathcal{U}$ is a biholomorphism between $\mathcal{U}$ and its image $\pi^{(p)}(\mathcal{U})$; b) $\tilde{f}\left(\pi^{(p)}(\mathcal{U})\right)$ does not intersect $\left\{\prod_{j=n-k+1}^{n} z_{j}=0\right\}$ (a sufficiently small neighborhood $\mathcal{U}$ surely satisfies both requirements). Then, we may consider the map

$$
f: \mathcal{U}_{1}=\mathcal{U} \cap \mathcal{E}_{(p)}^{n} \rightarrow \mathcal{U}_{2}=f(\mathcal{U}) \cap \mathcal{E}_{(p)}^{n}, \quad f \stackrel{\text { def }}{=} \pi^{(p)-1} \circ \tilde{f} \circ \pi^{(p)} .
$$

By construction, $f$ is a local automorphism of $\mathcal{E}_{(p)}^{n}$ and its associated automorphism of $\operatorname{Aut}\left(B^{n}\right)$ is $\tilde{f}$. By the hypotheses on $\tilde{f}$ and by Proposition 3.3, $f$ cannot extend to a global automorphism of $\mathcal{E}_{(p)}^{n}$.

\section{REFERENCES}

[Al] H. Alexander, Holomorphic mappings from the ball and polydisc, Math. Ann. 209 (1974), 249-256. MR0352531 (50:5018)

[DS] G. Dini and A. Selvaggi Primicerio, Localization principle of automorphisms on generalized pseudoellipsoids, J. Geom. Anal. 7 (4) (1997), 575-584. MR1669231 (99m:32032)

[FR] F. Forstnerič and J.-P. Rosay, Localization of the Kobayashi metric and the boundary continuity of proper holomorphic mappings, Math. Ann. 279 (1987), 239-252. MR919504 (89c:32070)

[KLS] K.-T. Kim, M. Landucci and A. Spiro, Factorization of proper holomorphic mappings through Thullen domains, Pacific J. Math. 189 (2) (1999), 293-310. MR 1696125 (2000e:32021)

[KS] K.-T. Kim and A. Spiro, Moduli space of ramified holomorphic coverings of $B^{2}$, in "Complex geometric analysis in Pohang (1997)", pp. 227-239, Contemp. Math., 222, Amer. Math. Soc., Providence, RI, 1999. MR.1653055 (99m:32033)

[La] M. Landucci, On the proper holomorphic equivalence for a class of pseudoconvex domains, Trans. Amer. Math. Soc. 282 (2) (1984), 807-811. MR732122 (85a:32033)

[LS] M. Landucci and A. Spiro, Proper holomorphic maps between complete Reinhardt domains in $C^{2}$, Complex Var. Theory Appl. 29 (1) (1996), 9-25. MR1381999 (97a:32028)

[Pi] S. Pinčuk, The analytic continuation of holomorphic mappings, Math. USSR Sb. 27 (1975) 375-392 (translation from Mat. Sb. (N.S.) 98 (140) (1975), 416-435). MR0393562 (52:14371)

[Pi1] S. Pinčuk, Holomorphic mappings of real-analytic hypersurfaces, Math. USSR Sb. 34 (1978), 503-519. MR 496595 (80c:32022)

[Ru] W. Rudin, Holomorphic maps that extend to automorphisms of a ball, Proc. Amer. Math. Soc. 81 (1981), 429-432. MR597656 (82c:32012)

[Ve] E. Vesentini, Capitoli scelti della teoria delle funzioni olomorfe, Unione Matematica Italiana, Bologna: Pitagora Ed., 1984.

[We] S. Webster, Biholomorphic mappings and the Bergman kernel off the diagonal, Invent. Math. 51 (1979), 155-169. MR.528021 (81e:32029)

Dip. Matematica Applicata "G. Sansone", Università di Firenze, Via di Santa Marta 3, I-50139 Firenze, ITALY

E-mail address: mario.landucci@unifi.it

Dip. Matematica e Informatica, Università di Camerino, Via Madonna delle Carceri, I-62032 CAmerino (Macerata), Italy

E-mail address: andrea.spiro@unicam.it 